\title{
Consideraciones sobre Cesáreas Practicadas en el ICSS
}

\author{
Pir el doctor José Ofman \\ Medico de la Universidad de Chile. Ex-residente de Maternidad del \\ Hospital Alvear de Buenos Aires. Obstetra del ICSS. \\ INDICACIONES DE LA VIA ALTA EN OBSTETRICIA \\ Evolución Histónica
}

Ya 700 años antes de Cristo, en la Lex Regia de Numa Pompilio figura la when de "abrir el vientre y extraer el feto cuando la madre ha muerto".

En la mujer viva fue practicada por el carnicero Nufer en el año de 1500. In 1610 el cirujano Trautmann lo hizo en una mujer viva dejando el útero Herte dentro del abdomen.

segun estadisticas de los sigles xwi, xwin y xix los resultados fueron los sizuentes: en 1600, Guillemau da el 100\% de mortalidad matema; en 1700,

- 1. lipau le asigna el 55\%; en 1750, Baudeloque 57\%; Kayser el 62\%; en 1820 , Melusir da otra vez el 100\%; en 1852, Caseaux presenta nuevamente una mor t.hlisd de $62 \%$.

Cim la asepsia y antisepsia y la sutura del útero después de la histerotomía n.disada en 1882 por Kehrer y Sanger, el indice de mortalidad materna baja $.115 \%$ (Credé) y al 28\% (Caruso).

Para alcjar los peligros de peritonitis post-cesárea se idearon diferentes téc nuas para abordar al útero extraperitonealmente, pero los resultados fueron rel.11ivon.

Ia historia moderna de la histerotomía se inicia con el descubrimiento de l. sulfoirogas que se usaron localmente dentro del abdomen, en la última dé4.rd.ı, al reducir casi a cero las posibilidades de infección.

Las indicaciones las podemos dividir en absolutas y relativas. Absolutas: placenta baja oclusiva y reducción acentuada de la pelvis ya sea por un vicio - pelviano, por un tumor previo, atresia de las partes blandas. Las relativas, gracas a su benignidad se han extendido a todos los capítulos de la obstetricia.

Hoy podemos decir que la cesárea está indicada en todas aquellas situaciones en que siendo muchos los riesgos de la exeracción por las vías naturales, la 
prosecución de la preñez o del parto significan una amenaza de muerte para la madre o para el feto. Asegurando así el mayo: porcentaje de éxitos a la madre y a su niño.

Las indicaciones actuales de la histerotomía de ninguna manera desaloja las otras operaciones que son clásicas en obstetricia.

Si para el niño se obtiene un resultado mejor, que el de cualquiera de las interienciones bajas, la madre corre riesgos que no se deben desconocer. Pero también es cierto que se han reducido al mínimum, por las técnicas operaterias. por los antibioicos y por las anestesias indicadas correctamente.

Analizando los resultades obtenides con diferentes recnicas de histeroto mias, se ve la superioridad de la segmentaria trin jeritoneal.

\section{Consideraciones sobre casáreas practicadas en el ICSS}

Sobre un total de 13.618 partos atendidos por el ICSS se han practicado 432 cesáreas li, que nos da $3.1 \%$, porcentaje muy parecido a las estadísticas de otras clínicas. De dicho número se estudiaron unicamente 204, por ser las más completas. Totlas ellas fueron trasperitoneales.

Grado de pureza y mortalidad materna.-1 a dividiremos en 4 grados de pureza, siguiendo la clasificacion de Sureau. Consideramos únicamente la placenta en el grado I de pureza ya que a cada paciente se le han practicado innumerables tactos. Según se observa en nuestros casos la mortalidad materna por peritonitis ha sido del $0 \%$. (Ver esquema No. 1).

Sobre 204 cesáreas transperitoneales la mortalidad materna global fue del $2.45 \%$.

Las pacientes fallecidas fueron:

1.-Caso No. 26. B. M. A. c. No. 01-0922 5, primipara a quien se le prac ticó una cesárea transperitoneal por despreporcion céfale pélvica. Presenta un shock obstétrico durante la intervención y fallece en la sala.

2.-Caso No. 203. R. M. c. No. 01-106855, tercera gestación, 1 parto pre maturo y un aborto. Embarazo de $71 \frac{1}{2}$ meses, se practica una cesárea transperi toneal por carditis reumática activa descompensada. Fallece el dia siguiente de la intervención.

3.-Caso No. 74. C. G. A c. No. 01-010986, multipara, dos embarazos normales, se le practica una cesárea segmentaria transperitoneal por despropor ción céfalo-pélvica, fallece en la sala por shock hemorrágico, por deficiencia en la técnica operatoria.

4.-Caso No. 123. R. C. c. No. 01-104193, multípara de 10, se le practica cesárea transperitoneal por placenta previa marginal. La enferma entra a la sala en shock hemorrágico, se opera con local; fallece al cierre de la pared.

5.-Caso No. 165. V. M. J. c. No. 01-013085, multípara, I embarazo nor- 


\section{ESQUEMA NUMERO 1}

Grado de pureza y mortalidad materna.

$\begin{array}{rcc}\text { Grupo } & \text { Totales } & \begin{array}{c}\text { Mortalldad Rlobal por sepsis } \\ \%\end{array} \\ \text { I } & 14 & 0 \\ \text { II } & 70 & 0 \\ \text { III } & 62 & 0 \\ \text { IV } & 1 & 0\end{array}$

\section{ESQUEMA NUMERO 2}

Gradı de pureza y morbilidad materna.

\begin{tabular}{rrrrr} 
Grupo & Totales & Normal & POST-OPERATORIo \\
Subfebril & Febril \\
I & 14 & 10 & 3 & 1 \\
II & 70 & 55 & 8 & 7 \\
IV & 62 & 48 & 12 & 2 \\
& 1 & & & 1 \\
& 147 & 113 & -23 & 11 \\
\hline
\end{tabular}

\section{ESQUEMA NUMERO 3}

Grado de pureza y morbilidad materna determinado en tanto por ciento.

\begin{tabular}{|c|c|c|c|c|}
\hline Grupo & Totales & $\begin{array}{c}\text { Normal } \\
\%\end{array}$ & $\begin{array}{c}\text { Subfebril } \\
\%\end{array}$ & $\begin{array}{c}\text { Febrll } \\
\%\end{array}$ \\
\hline I & 14 & 71.5 & 21.4 & 7.1 \\
\hline II & 70 & 78.6 & 11.4 & 10. \\
\hline 111 & $6: 2$ & 77.4 & 19.4 & 3.2 \\
\hline IV & 1 & & & \\
\hline Morbil & otal promedio & 76. & 15.3 & 8.7 \\
\hline
\end{tabular}




\section{ESQUEMA NUUMERO 4}

\section{Indicaciones de las Cesáreas en el ICSS.}

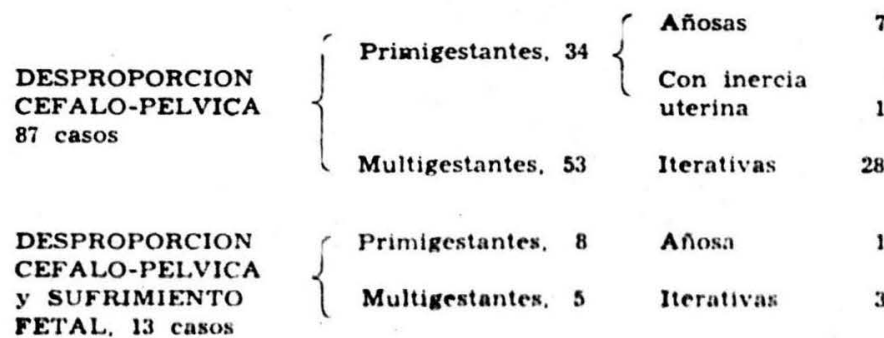

\section{SUFRIMIENTO \\ FETAL \\ 16 casos}

PLACENTA

PREVIA

24 casos

TOXICOSIS

GRAVIDICA

25 casos.

INERCIAS

UTERINAS

6 casos.

PRESENTACION

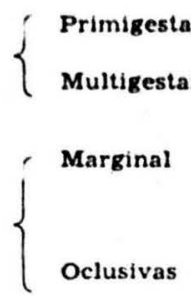

3

$\left\{\begin{array}{l}\text { Parciales } \\ \text { Totales }\end{array}\right.$

6

$15\left\{\begin{array}{l}\text { Primigestantes } \\ \text { Multigestantes }\end{array}\right.$

1

2

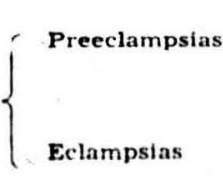

Cara

Frente

Situacion

transvers:

Primarias

Secundarias
7

1

28

3

Primigestantes 7

Multigestantes 2

$16\left\{\begin{array}{l}\text { Primigestantes } 12 \\ \text { Multigestantes }\end{array}\right.$

1

5

$10 \begin{cases}\text { Primigestantes } & 3 \\ \text { Multigestantes } & 7\end{cases}$

$5 \begin{cases}\text { Añosas } & 3 \\ \text { Gemelar } & 2\end{cases}$

1

DISTOCIAS PARTES BLANDAS

6 casos

PROCIDENCIA DEL CORDON

4 casos

DESPRENDIMIENTO DE PLACENTA

NORMOINSERTA 2 casos.

TBC pulmonar evolutiva

Carditis reumá-

tlea activa 
mal; se practica una cesárea segmentaria transperitoneal por eclampsia. Continúa con ataques eclámpticos subintrantes en el puerperio. Fallece al segundo dia.

Como vemos, la cesírea en si, no puede invocarse como causa de muerte siti) en un caso lo que reduce la mortalidad materna al $0.48 \%$.

Mortalidad infantil.-Presenta un 5.3\% de mortalidad infantil que no se preale culpar a la cesarea en si.

Morbilidud materna.-En todos los casos fuc usada la penicilina. Sólo se wiudiaron 147 casos por no tener los demás datos sobre el post-operatorio en cuinto a la temperatura. Si analizamos los esquemas Nos. 2 y 3, llegamos a la Conclusión de que la morbilidad es sensiblemente igual en los 3 primeros grupos. Alyora analizando la morbilidad total, resulta innegable que con el empleo de InN antibioticos solamente 13 fueron febriles lo que reduce a $8.7 \%$. Al estudiar lisi cesáreas antes de los antibióticos, observamos que los puerperios febriles asciepiden al $34.11 \%$.

Anestesias empleadas.-En las 204 enfermas operadas, dos se hicieron con f. $x .1(0.9 \%), 19$ con raquianestesia $(8.3 \%), 4$ con anestesia general, éter, $1.9 \%)$, y $179(88.9 \%$ ) con Ciclo-Oxigeno, Ciclo-Eter-Oxigeno, Ciclo-Pentotal( ) igeno.

La elección del anestésico en la gran mayoría de los casos se ha hecho sin . I estudio previo de la paciente. Hoy día es indispensable que el anestesista de l.h maternidades, esté íntimamente ligado a los servicios donde actúe, conozca l. patologia del embarazo; el tocólogo debe informarle en detalle la patología de - grivida, ya que sólo la colaboración inteligente entre ambos, podrá decidir la mestesia que el case requicere.

Comtrariamente de lo que se observa en las grandes maternidades nortemoricanas donde el $75 \%$ de las intervenciones se efectúan con raquianestesia, " in forma única, fraccionada o caudal. Nosotros la empleamos sólo en un 3\%. Muchos la han abandonado por los fracasos y accidentes que se han presentaclo. Según estudios de Babcock se considera que la peligrosidad de la rayuianestesia no está en el procedimiento sino en la manera de proceder de la prersona que la administra. Este es un nuevo capítulo que podríamos estudiar.

Preoperatorio.-Las enfermas, salvo en algunos casos de ancmias por hemorragia de placentas bajas, se operan sin transfusión y sin preparación alguna. les cirujanos generales usan gencrosamente la transfusión en los casos quirúr¿ace; es incomprensible que scamos nersotros los tocólogos tan poco adictos al - de la transfusión previa y durante la operación en una tan sangrante como "la cesárea.

En nuestros casos se ha usado la transfusión únicamente en dos:

1.- Pérdida abundante de sangre por mala retracción uterina.

2.-Shock obstétrico. 
Operacion.-En un solo caso se opero segun la operacion cesirea corporal (cesárea clásica). en los demás se practico la operacion cesáre segmentari: transperitoneal.

Tiempos operatorios.-La operación (paciente en decúbito-dorsal; mesia en posición de Trendelenburg: eracuación de la vejiga) se divide en los siguicntes ticmpos:

1.-Incision de !a pared del abdemen en una medida de 15 cme. (incision

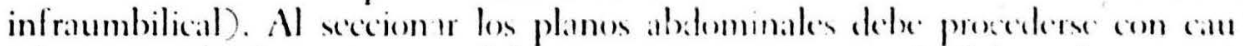

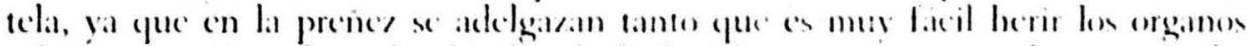
subvacentes. Se cubren len bordes de la herida con compresan de gassi. Aisla miento con compresis. (e'n ralmente un separaler bisalso da un buen campo.

2.-Incision y desprendimiento del peritonen uterino. A 2 cmts. mas o me nos hasta donde sube la veigga se secciona trans ersalmente el peritoneo en una longitud de 12 cmts. aprosimadamente. Se desprenden las adherencias naturales del peritóneo visceral que lo unen a la pared segmentaria.

3.-Incisión del segmento inferior-Se practica un ojal en el segmento y posteriormente se disocia a se secciona transversilmente de segmento.

4.- Extraccion del feto $)$ de los ancexos. Las membranas pueden quedar integras, se rempen dejande salir el liquide amiotion que se succiona con un aspirador electrico. Extracción del feto. Inveceion en el musculo uterino de ocits sicos. Alumbramiento esportáneo o manual de la placent:

5.-Cierre del útero.- En dos planos. El primero mís proximo a la cavidad (sutura continua), el segunde que cubre el anterior y afronta la pared restante (sutura continuas o por puntos separados). Posteriormente se rehate el peritonce, afrontando y uniendo sus bordes.

6.-Cierre del abdomen. En tres planos. Se extracon las compresis y el se parador, se verifica la resacción del útero y se procede a afrontar el peritoneo (sutura continuas), la apencurosis (sutura comtinua o por puntes separados) y l:i picl.

Pestoperaterion.-. En tedos los casos se usi la penicilina en forma profilictica. En el 14.7\% de los cases se usie con combinacion de lat estreptomicina. Las otras drogas de use corricute fucron: prostigmine, ergotrate, vitamina C, plasmal, 1)extrosa al $5 \%$ en wheion salina 0 en agua. In los casos de celampsia y precelampsia se agrege el trat amiento dásico para coses casos: sulfato de magnesia, dextrosa hipertónica al $33 \%$ y $50 \%$, barbitúricos.

Indicaciones de las cesareas. Sógún nuestro estudio (espucma No. 4), se obscrva yue 100 enferme presentaron desproporcion pelvicofetal que nos da un porcentaje global del t'y, Subdividimos on 2 grupos: desproporcion cefalo-

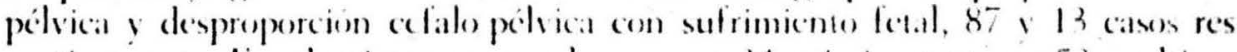
pectivamente. Lin el primer gripo observamos it primigestantes y 53 mulagestantes. De las primigestantes 7 de cllas anosis. Ina de cllos (case No. 12) presenta además una inere a secu adaria. Vemos que en los casos No. 16, 17 y 33 hubo intento de forceps. 
De las multigestantes, 28 son iterativas; de cllas hay 8 asose en que no at. bien justificada la intervención.

Ilos los antibioticos han bencliciado la conduccion del parto en la estre. dhe pelviana que nos permite ampliar el empleo del parto de prueba y de la "yectacion, además ya no existen las limitaciones impucstas por el concepto de com impuros para indicaciones posteriores del parto yuirurgico. Según estudios Le. Len por el doctor Manuel Aviles. Maternidad del Salvador, Santiago de 1 hale, solere 315 pacientes los partos posteriores a cesarea fueron: espontaneos 6. $04 \%$, operatorios por via vaginal $118.13 \%$ y únicamente el $25.88 \%$ por via "ieraton io ablominal. Coinciden con estas ideas Stander. Water, Wilson. El mumere de cesareas itcrativas es la consecuencia logica de los factores que ordin.rimente motivan su indicación, pudiendo agregarse a ellas, la poderosa inllacencia del antecedente de una histerotomía previa que seguramente superbloramos en nuestras determinaciones.

De esto podemos deducir que existiendo una pelvis límite y dinámica norm.1 está indicada la prueba de trabajo que muchas veces nos dará la solución d.l problema. Podría haberse intentado en los casos arriba enumerados. En 8 huly intento de forceps ${ }^{1}$.

Revisando las actas quirúrgicas de las enfermas de cesáreas iterativas ve-

yue la cicatriz operatoria anterior es perfectal, exceptuando en un caso (1. 5t) en que hay gran cantidad de adherencias fibrosas. En algunos casos whervaron adherencias laxas en cl fondo uterino.

In 13 casos fuera de la desproporción presentaron sufrimiento fetal que mulica plenamente la intervención por vía alta.

In 16 casos $(7.8 \%$ ) observamos que la únca causa de intervención fue 1 1 utrimicnto fetal.

Por placenta previa 24 casos ( $11.8 \%$ ), observamos las siguientes variedaa. marginal (3 casos) en que la placenta llege al orificio interno, todas en mulupiras. (O)lusivas (2l casos), parciales (6 casos) en que la menor parte de condedones han pasade al segmento inferior del lado opuesto, todas en mul

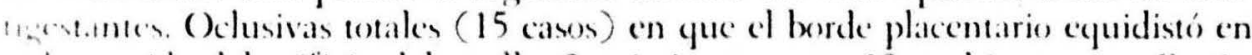
tente sentido del orificio del cuello. 2 primigestantes y 13 multigestantes. Según "au 110 cases intervenidos la gran mayoría corresponde a enfermas multigestan 11. 8 de cllas de 5 o más partos.

la operación cesárea abdominal en la terapéutica de la placenta baja fue umlizala por primera vez por Krocning y Sellheim (1908) (hoy se realiza entre 1 30\% y $63 \%$ de los casos, scgún estudios de II. (ooceker). Da un porcentaje nunimo de fracasos maternos, como el mejor de los otros tratamicntos (O\% de montalidad, 1)e Lee; 3.05\%, (iuggisberg; 3.4\%, Panhow); y al discreto de muer $\therefore$ Actales (7\%, Pankow; 8.33\%, D. Iee, 10\%, Walthard), se agregan otras

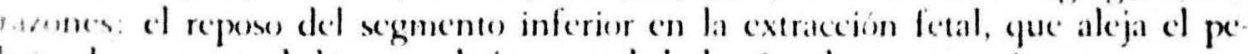
lige de rotura y el de poner bajo control de la vista la zona uterina que sangra.

1. Re han incluldo casos clínicos incompletos por wer de Interín para el exturio $y$ con el fin te hacer una critica constructiva para el futuro, ya que el ICSS tiene interéx en hacer una entadiatica atil. 
En uno de los casos (No. 132) la etiología puede ser la fibromatosis múltiple; en los demás no tenemos ningún antecedente en la historia clínica. Se registró una muerte materna lo que nos da $4.2 \%$ de mortalidad materna y 5 fetales (20.7\%). El aumento de muerte fetal en relación con las estadísticas de otras clínicas se puede deber a que son generalmente gente de condiciones sociales bajas y que ingresaron en la clinica después de sangrar abundantemente en sus casas.

Por toxicosis gravidica 25 casos (12.3\%); preeclampsias (9 casos) de las cuales 7 en primigestantes y 2 en multigestantes. Eclampsias (16 casos), 12 en primigestantes y 4 en muligestantes. Dos entermas fueron operadas en coma (No. 141 y $1+3)$. Una de las enfermas presentó hemorragia meníngea. Una enferma fallece por continuar con ataques eclámpticos subintrantes (caso No. i65). Al revisar dichas enfermas se observa que no han tenido control prenatal previo. Muchos de estos casos se podrían prevenir obligando la asistencia prenatal de toda enferma atendida en la clínica.

Por presentación de cara 1 caso $(0.5 \%)$.

Por presentación de frente 5 casos (2.5\%). En un caso se comprobó que la causa etiológica de la presentación de frente se debió a un meningocelo (No. 168). En un caso se intentó forceps y en otro caso mutarlo a vértice o cara.

Por presentación transversa 10 casos $(4.9 \%)$. Cinco de las enfermas ingresaron con procidencia del brazo y una con procidencia del cordón. Todas las enfermas presentaron la bolsa rota.

Por inercia uterina 6 casos $(2.9 \%)$, tres eran primigestantes añosas y otro una inereia uterina secundaria (No. 187), dos con embarazo gemelar.

Por distocia de las partes blandas 6 casos $(2.9 \%)$.

Por procidencia del cordón 4 casos (1.9\%). En dos de los casos se puede considerar como causa etiolígica la multiparidad que influye por la hipotonía del útcro; las enfermas Nos. 198 y 199 que han tenido 11 y 5 partos normales respectivamente. Pedemos considerar en estos casos que la intervención fue oportuna y feliz ya que obtuvimos todos los fetos vivos. Cieneralmente el pro medio de mortalidad fetal es del 35\% cuando los fetos están vivos antes de la intervención (32\%, H. Sicgmund; 32\%, Halter; 36\%, Martius; 37.5\%, Zwiefel; $37.9 \%$, Sichwetzer).

Por desprendimiento mínimo de placenta normoinserta 2 casos $(0.98 \%)$. Nosotros hemos obtenido un éxito completo (el número es tan pequeño que no nos deja sacar conclusiones) y ello puede abedecer a la intervención inmediata. Consultando estadisticas observamos que hoy todavia mantiene su gravedad. (Ya que presenta la siguiente mortalidad materna: $3.1 \%$. Pankow; $3.78 \%$. So lomons; 4.6\%, A. Brindeau). El pronóstico fetal es más grave, esta mortalidad gira alrededor de $73 \%$ (56\%, Lieven; $62 \%$, Frankl; $76 \%$, Pankow; 77,8\%, Barchet).

Por otras causas 3 casos ( $1.52 \%$ ). Una enferma por TBC pulmonar (No. 202). Una por carditis rcumática activa descompensada (No. 203) y una por reconstrucción del periné (No. 204). 
En un caso (No. 171) al abrir el abdomen se lesiono el intestino delgado. Sc suturó transicersalmente. La enferma evoluciona normal y afcebril.

Segun nuestro estudio observamos que la cesirea ha centrado en la posibi lided terapéutica de gran parte de los capitulos de la obstetricia desde el cm. pleo de los antibióticos. Pero en el tocologo de hoy, como en el de siempre, delx pucvalecer al criterio clinico que $k$ indicara el momento más oportuno para

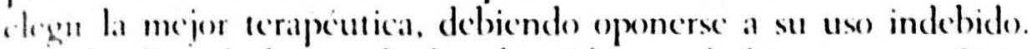

Analizande les resultados obtenidos con la histerotomia clásica, la suprasim Im.tra transperitoneal, la extraperitoneal por artificio, observamos la superio whis pronóstica de la histerotomía segmentaria transperitoneal que nos arroja . I $\omega^{\prime}$ i de mortalidad materna séptica. Este porcentaje ideal comparado con el major obtenido con la extraperitoneal pura $(0.5 \%)$ nos indica que dicha téenia) en hoy por hoy la más indicada; a ello se le suma la simplicidad de su técnica que la coloca al alcance de todo médico obstetra. 